\title{
Nivel de Conocimiento sobre Pasta Dental Fluorada en Padres y Profesores de Preescolares
}

\author{
Level of Knowledge about Fluoride Toothpaste \\ in Parents and Teachers from Preschool Children
}

\author{
Manuel Antonio Mattos-Vela*,*; Milagros Bertha Carrasco-Loyola* \& Suelen Giuliana Valdivia-Pacheco*
}

MATTOS-VELA, M. A.; CARRASCO-LOYOLA, M. B. \& VALDIVIA-PACHECO, S. G. Nivel de conocimiento sobre pasta dental fluorada en padres y profesores de preescolares. Int. J. Odontostomat., 7(1):17-24, 2013.

RESUMEN: El objetivo del estudio fue determinar el nivel de conocimiento sobre pasta dental fluorada según la fuente de información sobre flúor en padres y profesores de preescolares. Se aplicó un cuestionario a una muestra representativa de padres y a todos los profesores de preescolares de instituciones educativas iniciales estatales del distrito de la Molina, Lima, Perú. El nivel de conocimiento se categorizó en tres niveles: bajo (0-2 puntos), medio (3-5 puntos) y alto (67 puntos). Se utilizó tablas de distribución de frecuencias para el análisis univariado y las pruebas chi cuadrado y exacta de Fisher para el análisis bivariado. El nivel de conocimiento sobre pasta dental fluorada más frecuente fue el nivel medio tanto en los padres ( $n=68,45 \%$ ) como en los profesores $(n=52,58,4 \%)$. El ítem: el cepillado de dientes con pasta dental con flúor puede curar la caries inicial, presentó el menor número de aciertos en su respuesta con 50 (33,1 \%) y 19 (21,4 \%) padres y profesores respectivamente. Cuando se relacionó el nivel de conocimiento con las fuentes de información sobre flúor se encontró que en el caso de los padres, la charla educativa $(p=0,014)$ y el dentista $(p=0,003)$ estaban asociados, mientras que en el caso de los profesores, solo se halló asociación estadísticamente significativa con la charla educativa ( $p=0,013)$. El nivel predominante de conocimientos sobre pasta fluorada en los padres y profesores fue el nivel medio y estuvo asociada al dentista y la charla educativa como fuentes principales de información.

PALABRAS CLAVE: dentífricos, fluoruros, salud bucal.

\section{INTRODUCCIÓN}

A lo largo de la historia de la humanidad se ha identificado a la caries dental como una enfermedad omnipresente y con alta prevalencia afectando a la mayor parte de la población mundial. Como tal, puede ser controlada a través de medidas preventivas (Pérez, 2009) y ser tratada lo más tempranamente posible; ya que se conoce que las lesiones cariosas iniciales pueden ser controladas y revertidas (Meier \& Jensen, 1984; Lussi, 1993).

En la actualidad, la caries dental es considerada un problema de salud pública en muchos países; por lo cual resulta de vital importancia la educación y promoción de la salud bucal, así como garantizar un mayor acceso a los servicios de salud que permitan mejorar la salud de la población (Margolis et al., 2001; Arblaster et al., 1996).
El uso del flúor, es la medida preventiva más difundida y con mayor repercusión en la prevención de la caries dental en la población. Sin embargo, el flúor debe considerarse como un medicamento, y como tal, contar con la supervisión del médico pediatra, médico de familia u odontopediatra para evitar su empleo de manera indiscriminada (Barbería et al., 2005).

Los agentes fluorados de uso tópico son ampliamente comercializados y están a disposición del público en supermercados y farmacias ya que, algunos de ellos, se acogen a la legislación de los cosméticos, lo que permite una gran accesibilidad a estos productos, sin considerar sus contraindicaciones y riesgos. Es conocido que el flúor puede producir toxicidad crónica conduciendo a la fluorosis dental, que deriva

* Cirujano Dentista, Facultad de Odontología, Universidad de San Martín de Porres (USMP), Lima, Perú.

"Magister en Estomatología, Facultad de Odontología, Universidad de San Martín de Porres (USMP), Lima, Perú.

Financiamiento: Compartido entre los autores, Facultad de Odontología de la Universidad de San Martín de Porres. 
de la ingestión continua de pequeñas dosis de fluoruros pero que son suficientes por su efecto acumulativo (Barbería et al.).

Los estudios indican que el flúor que previene la caries dental es aquel que está constantemente presente en la cavidad bucal (Forward, 1980; Mellberg, 1991). Esto implica que la población debe presentar hábitos de higiene bucal que incluya el uso de dentífricos fluorados.

Por otro lado, los dentífricos comercializados como infantiles, no necesariamente contienen baja concentración de flúor, se caracterizan principalmente por su sabor y presentación distintos, lo cual puede motivar su ingestión en niños pequeños (Barbería et al.), a quienes se les considera como grupo de riesgo para fluorosis dental. En ese sentido, la promoción de la salud bucal, debe ser prioridad para un buen cuidado odontológico en el infante, procurando educar a los padres, profesores, otros profesionales de la salud y todos aquellos que influencian a la población de mayor riesgo; promoviendo así el mantenimiento de hábitos saludables (Contreras et al., 2008). La educación, promoción y buenas prácticas en salud bucal son trascendentales y no representan un alto costo, en comparación a los tratamientos restauradores, sobre todo para países en vías de desarrollo, donde las poblaciones marginadas y en condiciones de pobreza son las más afectadas (Delgado et al., 2006).

Se ha sugerido la intervención educativa como un método eficaz y certero para obtener e incrementar conocimientos sobre salud e higiene bucal (Contreras et al.), además posibilita que los niños e infantes, que participen de estas intervenciones, transmitan lo aprendido al colectivo y la familia, con lo cual se convierten en verdaderos promotores de la salud (Crespo et al., 2010).

Son escasas las investigaciones sobre el nivel de conocimientos de los padres y profesores sobre pastas dentales infantiles fluoradas. En China, un estudio encuentra que un $70 \%$ de estudiantes de diferentes grados, padres y profesores conocen el efecto preventivo de la pasta dental fluorada. Padres y profesores, $93 \%$ y $56 \%$ respectivamente, reconocieron su importante rol en promover la salud bucal (Liu et al., 2007). En una zona rural de Australia se reportó que muchos de los padres consideraron que la pasta dental fluorada reduce el riesgo de caries, pero no saben si se debía usar en infantes (Gussy et al., 2008).
El objetivo del estudio fue determinar el nivel de conocimiento sobre pasta dental fluorada según la fuente de información sobre flúor en los padres y profesores de preescolares de instituciones educativas iniciales estatales del distrito de La Molina.

\section{MATERIAL Y MÉTODO}

Se trata de un estudio descriptivo de corte transversal el cual es parte integrante de un estudio mayor que buscó evaluar las características de los dentífricos empleados por los preescolares y las prácticas (de preescolares, sus padres y profesores) y conocimientos (de los padres y profesores) sobre uso de pasta dental fluorada.

Población y Muestra. La población estuvo conformada por los responsables (padre, madre u otro) de 1183 niños de 3 a 5 años de edad matriculados en el 2010 en ocho instituciones educativas iniciales estatales del distrito de La Molina, Lima-Perú y por 98 profesores y auxiliares de estas instituciones: Oscar Bravo Ratto ( $\left.N^{\circ} 130\right)$, Virgen de Fátima ( $\left.N^{\circ} 121\right)$, Virgen del Carmen ( $\left.N^{\circ} 112\right)$, Viña Alta ( $\left.N^{\circ} 140\right)$, Cuna Jardín (CJ) Viña Alta, Unión Latinoamericana ( $\left.N^{\circ} 1235\right)$, Institución Educativa Municipal (IEM) Descubriendo y CJ Santa Rosa de Lima.

Para determinar el tamaño muestral se consideró un valor máximo de la proporción de la variable de estudio $(0,5)$ puesto que el estudio consideró muchas variables, el nivel de confianza fue de $95 \%$ y el error de precisión 0,07 . El tamaño mínimo de muestra fue 169 a lo cual se añadió un 15\% considerando las no respuestas, obteniéndose una muestra de 195 padres. Se empleó un muestreo aleatorio sistemático con probabilidad proporcional al tamaño de las instituciones educativas. En el caso de los profesores se trabajó con toda la población.

Los criterios de exclusión fueron: padres de niños con impedimentos físicos o mentales, que dejaron de asistir a clases, con datos incompletos o incoherentes en su ficha de datos personales (respecto a la edad y sexo) y que no contaron con la respectiva autorización.

Los criterios de inclusión para los profesores y auxiliares de los preescolares fueron que realicen una labor educativa directa con los niños y que tengan un vínculo laboral formal con la institución educativa inicial en la que laboran. 
Técnica e instrumentos de recolección de datos. Se empleó un mismo cuestionario para estudiar el nivel de conocimientos sobre pasta dental con flúor en los padres y profesores de los preescolares. Este cuestionario fue adaptado de un estudio realizado en China (Liu et al.). La validación del instrumento se realizó a través del juicio de expertos y una prueba piloto, además del índice de Kuder Richardson (para evaluar la confiabilidad interna de estos puntos, obteniéndose un resultado de 0,99). El cuestionario constó de ocho ítems; siete de ellos, midieron los conocimientos sobre pasta dental fluorada y el octavo, la fuente de información sobre el flúor. El nivel de conocimientos se obtiene sumando el puntaje obtenido luego de responder las declaraciones sobre conocimiento de flúor. La respuesta correcta tuvo un valor de uno y la incorrecta de cero, subsecuentemente se categorizó en tres niveles: bajo (0-2 puntos), medio (3-5 puntos) y alto (67 puntos). El proyecto fue aprobado por el Instituto de Investigación de la Facultad de Odontología de la Universidad San Martín de Porres.

Se solicitó el permiso de las autoridades de las instituciones educativas para la realización del estudio. Se encuestó primero a los profesores y auxiliares, los cuales llenaron y devolvieron el cuestionario al término de la misma, en las instalaciones de las instituciones educativas. A los padres de los preescolares se les envió la encuesta a través de sus hijos para devolverlo resuelto en los siguientes días. Luego que la persona entregaba el cuestionario lleno se le entregó un tríptico educativo que explicaba los temas abordados en el cuestionario.
El procesamiento y análisis de los datos se realizó empleando el paquete estadístico SPSS v15. Se utilizó tablas de distribución de frecuencias para describir todas las variables de estudio. Para el análisis bivariado se empleó la prueba de chi cuadrado y la prueba exacta de Fisher. Las pruebas fueron trabajadas a un nivel de significancia estadística de $5 \%$.

\section{RESULTADOS}

La muestra final estuvo constituida por 151 padres y 89 profesores de los niños preescolares, siendo la tasa de respuesta $77,5 \%$ y $90,8 \%$ respectivamente. Las madres $(80,8 \%)$ fueron quienes principalmente respondieron el cuestionario dirigido al responsable del niño, mientras que en el caso de los profesores todos fueron del sexo femenino (Tabla I).

El $91 \%$ de padres y profesores de los preescolares habían escuchado la palabra pasta dental con flúor. De los seis ítems restantes, tres de ellos fueron correctamente respondidos por aproximadamente tres cuartas partes de los padres y profesores encuestados: "la pasta dental con flúor es aquella pasta de dientes que contiene flúor", "el cepillado de dientes con pasta dental con flúor puede prevenir la caries" y "el cepillado de dientes con pasta dental con flúor puede aumentar la resistencia de los dientes a la caries". Los otros tres ítems fueron respondidos correctamente solo por menos de la mitad de los padres y profesores encuestados, siendo el ítem: "el cepillado de dientes

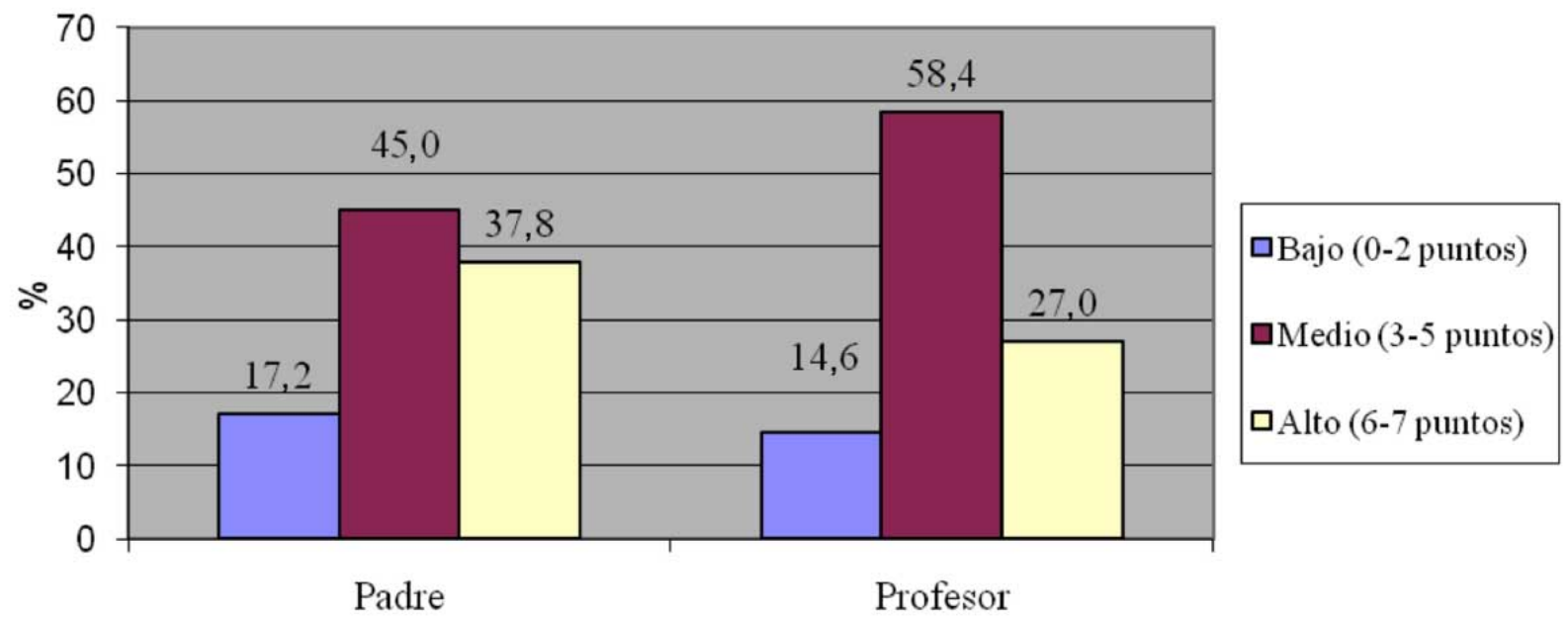

Fig. 1. Nivel de conocimiento sobre pasta dental fluorada en padres y profesores de preescolares. 
Tabla I. Características generales de padres y profesores de preescolares.

\begin{tabular}{|c|c|c|c|c|}
\hline \multirow{2}{*}{ Características generales } & \multicolumn{2}{|c|}{ Padre $(n=151)$} & \multicolumn{2}{|c|}{ Profesor $(n=89)$} \\
\hline & $\mathbf{n}$ & $\%$ & $\mathbf{n}$ & $\%$ \\
\hline \multicolumn{5}{|l|}{ Sexo } \\
\hline Masculino & & & 0 & 0 \\
\hline Femenino & & & 89 & 100,0 \\
\hline \multicolumn{5}{|l|}{ Institución educativa } \\
\hline Oscar Bravo Ratto & 30 & 19,9 & 20 & 22,5 \\
\hline Virgen de Fátima & 14 & 9,3 & 8 & 9,0 \\
\hline Virgen del Camen & 33 & 21,8 & 19 & 21,4 \\
\hline Viña Alta & 38 & 25,1 & 17 & 19,1 \\
\hline CJ. Viña Alta & 8 & 5,3 & 6 & 6,7 \\
\hline Unión Latinoamericana & 11 & 7,3 & 2 & 2,2 \\
\hline IEM Descubriendo & 11 & 7,3 & 8 & 9,0 \\
\hline CJ Santa Rosa de Lima & 6 & 4,0 & 9 & 10,1 \\
\hline \multicolumn{5}{|l|}{ Parentesco con el niño } \\
\hline Madre & 122 & 80,8 & & \\
\hline Padre & 23 & 15,2 & & \\
\hline Otro & 6 & 4,0 & & \\
\hline
\end{tabular}

con pasta dental con flúor puede curar la caries inicial", la que presentó menor número de aciertos en su respuesta con 50 $(33,1 \%)$ y $19(21,4 \%)$ padres y profesores respectivamente (Tabla II).

En cuanto al nivel de conocimiento sobre pasta dental fluorada fue más frecuente el nivel medio tanto en los padres $(n=68 ; 45 \%)$ como en los profesores $(n=52$; $58,4 \%$ ) (Fig. 1).

En la pregunta sobre la manera en que se obtuvo información sobre el flúor (en la cual se podía marcar más de una respuesta) se encontró que el dentista, seguido de los medios de comunicación masivos, fueron las fuentes más comunes tanto para los padres $(53,7 \%$ y $47 \%$ respectivamente) como para los profesores de los preescolares $(53,1 \%$ y $39,5 \%$ respectivamente) (Tabla III). En esta pregunta se trabajó con una muestra menor en los padres $(n=132)$ $y$ en los profesores $(n=81)$ ya que se des-

Tabla II. Conocimiento sobre pasta dental fluorada en padres y profesores de preescolares.

\begin{tabular}{|c|c|c|c|c|c|}
\hline \multirow{2}{*}{ Conocimiento sobre pasta dental fluorada } & & \multicolumn{2}{|c|}{ Padre } & \multicolumn{2}{|c|}{ Profesor } \\
\hline & & $\mathbf{n}$ & $\%$ & $\mathbf{n}$ & $\%$ \\
\hline \multirow[t]{2}{*}{ Ha escuchado la palabra "pasta dental con flúor" } & $\mathrm{Si}$ & 137 & 90,7 & 81 & 91,0 \\
\hline & Corr & 119 & 78,8 & 66 & 74,2 \\
\hline \multirow{3}{*}{$\begin{array}{l}\text { La pasta dental con flúor es aquella pasta de dientes que } \\
\text { contiene Flúor }\end{array}$} & Incorrecto & 2 & 1,3 & 4 & 4,5 \\
\hline & No sabe & 30 & 19,9 & 19 & 21,3 \\
\hline & Correcto & 113 & 74,8 & 65 & 73,0 \\
\hline \multirow{3}{*}{$\begin{array}{l}\text { El cepillado de dientes con pasta dental con flúor puede } \\
\text { prevenir la caries }\end{array}$} & Incorrecto & 6 & 4,0 & 5 & 5,6 \\
\hline & No sabe & 32 & 21,2 & 19 & 21,4 \\
\hline & Correcto & 50 & 33,1 & 19 & 21,4 \\
\hline \multirow{3}{*}{$\begin{array}{l}\text { El cepillado de dientes con pasta dental con flúor puede curar } \\
\text { la caries inicial (la caries que está comenzando) }\end{array}$} & Incorrecto & 51 & 33,8 & 40 & 44,9 \\
\hline & No sabe & 50 & 33,1 & 30 & 33,7 \\
\hline & Correcto & 106 & 70,2 & 64 & 71,9 \\
\hline \multirow{3}{*}{$\begin{array}{l}\text { El cepillado de dientes con pasta dental con flúor puede } \\
\text { aumentar la resistencia de los dientes a la caries }\end{array}$} & Incorrecto & 8 & 5,3 & 5 & 5,6 \\
\hline & No sabe & 37 & 24,5 & 20 & 22,5 \\
\hline & Corr & 71 & 47,0 & 41 & 46,1 \\
\hline \multirow{2}{*}{$\begin{array}{l}\text { La cantidad apropiada de flúor es buena para la salud, pero la } \\
\text { cantidad excesiva de flúor puede ocasionar daño a la salud }\end{array}$} & Incorrecto & 8 & 6,0 & 7 & 7,8 \\
\hline & No sabe & 71 & 47,0 & 41 & 46,1 \\
\hline \multirow{3}{*}{$\begin{array}{l}\text { La cantidad de pasta dental con flúor que se debe aplicar para } \\
\text { niños de } 3 \text { a } 6 \text { años de edad debería ser del tamaño de una } \\
\text { lentejita }\end{array}$} & Correcto & 63 & 41,7 & 41 & 46,1 \\
\hline & Incorrecto & 16 & 10,6 & 13 & 14,6 \\
\hline & No sabe & 72 & 47,7 & 35 & 39,3 \\
\hline
\end{tabular}


Tabla III. Fuente de información sobre flúor en padres y profesores de preescolares.

\begin{tabular}{lcccc}
\hline \multirow{2}{*}{ Fuente de información } & \multicolumn{2}{c}{ Padre $\mathbf{( n = 1 3 2 )}$} & \multicolumn{2}{c}{ Profesor $(\mathbf{n}=\mathbf{8 1})$} \\
\cline { 2 - 5 } & $\mathbf{n}$ & $\mathbf{\%}$ & $\mathbf{n}$ & $\mathbf{\%}$ \\
\hline Medios masivos & 62 & 47,0 & 32 & 39,5 \\
Charla educativa & 38 & 28,8 & 29 & 35,8 \\
Dentista & 71 & 53,8 & 43 & 53,1 \\
Amigos o familiares & 9 & 6,8 & 10 & 12,3 \\
Otra fuente & 9 & 6,8 & 3 & 3,7 \\
\hline
\end{tabular}

* Más de una respuesta permitida.

Tabla IV. Nivel de conocimiento sobre pasta dental fluorada según fuente de información en padres y profesores de preescolares.

\begin{tabular}{|c|c|c|c|c|c|c|c|c|}
\hline \multirow{3}{*}{ Fuente de información } & & \multicolumn{6}{|c|}{ Nivel de conocimiento } & \multirow[b]{3}{*}{$\mathbf{p}$} \\
\hline & & \multicolumn{2}{|c|}{ Bajo (0-2 puntos) } & \multicolumn{2}{|c|}{ Medio (3-5 puntos) } & \multicolumn{2}{|c|}{ Alto (6-7 puntos) } & \\
\hline & & $\mathbf{n}$ & $\%$ & $\mathbf{n}$ & $\%$ & $\mathbf{n}$ & $\%$ & \\
\hline \multicolumn{9}{|l|}{ Padres } \\
\hline \multirow[t]{2}{*}{ Medios masivos } & $\mathrm{Si}$ & 5 & 45,5 & 30 & 46,9 & 27 & 47,4 & 0,993 \\
\hline & No & 6 & 54,5 & 34 & 53,1 & 30 & 52,6 & \\
\hline \multirow[t]{2}{*}{ Charla educativa } & $\mathrm{Si}$ & 2 & 18,2 & 12 & 18,8 & 24 & 42,1 & $0,014^{*}$ \\
\hline & No & 9 & 81,8 & 52 & 81,3 & 33 & 57,9 & \\
\hline \multirow[t]{2}{*}{ Dentista } & $\mathrm{Si}$ & 2 & 18,2 & 30 & 46,9 & 39 & 68,4 & 0,003† \\
\hline & No & 9 & 81,8 & 34 & 53,1 & 18 & 31,6 & \\
\hline \multirow[t]{2}{*}{ Amigos o familiares } & $\mathrm{Si}$ & 2 & 18,2 & 2 & 3,1 & 5 & 8,8 & 0,103 \\
\hline & No & 9 & 81,8 & 62 & 96,9 & 52 & 91,2 & \\
\hline \multicolumn{9}{|l|}{ Profesores } \\
\hline \multirow[t]{2}{*}{ Medios masivos } & $\mathrm{Si}$ & 2 & 40,0 & 23 & 44,2 & 7 & 29,2 & 0,449 \\
\hline & No & 3 & 60,0 & 29 & 55,8 & 17 & 70,8 & \\
\hline \multirow[t]{2}{*}{ Charla educativa } & $\mathrm{Si}$ & 0 & 0 & 15 & 28,8 & 14 & 58,3 & $0,013^{*}$ \\
\hline & No & 5 & 100,0 & 37 & 71,2 & 10 & 41,7 & \\
\hline \multirow[t]{2}{*}{ Dentista } & $\mathrm{Si}$ & 4 & 80,0 & 28 & 53,8 & 11 & 45,8 & 0,435 \\
\hline & No & 1 & 20,0 & 24 & 46,2 & 13 & 54,2 & \\
\hline \multirow[t]{2}{*}{ Amigos o familiares } & $\mathrm{Si}$ & 1 & 20,0 & 7 & 13,5 & 2 & 8,3 & 0,520 \\
\hline & No & 4 & 80,0 & 45 & 86,5 & 22 & 91,7 & \\
\hline
\end{tabular}

* Diferencia significativa: Prueba exacta de Fisher

† Diferencia altamente significativa: Prueba de chi cuadrado

cartaron a los que refirieron no haber escuchado sobre pasta dental. Cabe resaltar que en el caso de la muestra de padres, varios no respondieron la pregunta de cómo obtuvieron información sobre flúor, a pesar de haber expresado que tenían referencias sobre aquel.

Cuando se relacionó el nivel de conocimiento con cada una de las fuentes de información sobre flúor se encontró que en el caso de los padres, la charla educativa y el dentista estaban asociados de manera significativa $(p=0,014)$ y altamente significativa $(p=0,003)$ respectivamente, mientras que en el caso de los profesores, solo se halló asociación estadísticamente significativa con la charla educativa $(p=0,013)($ Tabla IV). 


\section{DISCUSIÓN}

El estudio incluyó a ocho de los nueve centros de educación inicial públicos del distrito de La Molina, Lima Metropolitana. Una de las instituciones educativas no aceptó participar del estudio. La muestra de los padres de los preescolares fue representativa para la población de estudio. En el caso de los profesores, todos fueron incluidos en la investigación.

El estudio proporciona información importante sobre conocimientos acerca del uso de la pasta dental en preescolares. Recoge información no solo de los padres, sino también de los maestros. En Perú, los índices de fluorosis reportados alcanzan el 10,1\% (Ministerio de Salud, 2005) por lo que resulta importante conocer qué información maneja esta población que es la que guiará la conducta de los infantes en el uso de la pasta dental fluorada la cual podría reducir el riesgo a fluorosis.

La amplia difusión de los beneficios del uso de las pastas fluoradas y el mayor acceso que se tiene hoy en día a estos productos, promueve la utilización de las mismas en todas las edades. Sin embargo, el conocimiento que demuestran los padres y profesores es principalmente de nivel intermedio, lo que podría conllevar a practicas peligrosas en higiene bucal, que en lugar de mejorar la salud bucal pueden producir un detrimento de ella, ante un exceso de flúor. Un nivel alto de conocimiento fue más frecuente en el grupo de los padres que en los profesores. Esto puede explicarse debido a que los padres resultan estar mejor informados en estos aspectos de salud bucal por ser los más interesados en el bienestar de sus propios hijos y, es posible, que hayan tenido acceso a mayor información sobre salud oral con la crianza de sus otros hijos. Los resultados obtenidos en el grado de conocimiento de los padres es similar a lo reportado en un estudio realizado en Beijing (Liu et al.), donde los porcentajes de nivel de conocimiento medio y alto fueron de 45 y $32 \%$. En el caso de los maestros, los porcentajes encontrados son mayores a los del referido estudio, en los niveles de conocimiento medio $(58 \%$ y $47 \%$, respectivamente) y similar en el nivel alto ( $27 \mathrm{y}$ $29 \%$, respectivamente). Esta diferencia puede ser explicada en el hecho que en este estudio, los profesores fueron del nivel inicial, exclusivamente, mientras que en el estudio de Liu et al. eran profesores de primaria y secundaria, los que posiblemente, tienen menor acceso a este tipo de información.
Considerando que más del $90 \%$ de los padres y profesores se familiarizan con el término "pasta dental con flúor", solo cerca de tres cuartas partes de los padres y profesores, respectivamente saben lo que significa este término y una proporción menor de estos conocen sus beneficios. El efecto del flúor en las frases "prevenir caries" y "resistencia de los dientes a la caries" fueron las expresiones que obtuvieron los mayores porcentajes de respuestas correctas, lo que puede deberse a la coincidencia en los mensajes que publicitan las empresas productoras de pastas dentales. Los resultados obtenidos fueron similares a los de Gussy et al., en su estudio realizado en Australia, donde un $73,8 \%$ de los padres conocían que "el flúor previene la caries dental haciendo los dientes más fuertes" y un $66 \%$ respondieron que "el flúor en la pasta dental previene la caries dental".

La pregunta que obtuvo el menor porcentaje de respuestas correctas tanto en los padres como en los profesores fue la referida al efecto de las pastas dentales sobre las lesiones incipientes de caries, las respuestas incorrectas superaban al porcentaje de repuestas contestadas como "no sabe", lo que no ocurrió con el resto de las preguntas. La escasa difusión de esta información por los medios de comunicación y los profesionales de la salud, podría ser la razón de la alta proporción de respuestas incorrectas obtenidas. Esto refuerza la idea que es el profesional de salud el responsable de promover prácticas saludables en la población y el empleo de los medios de comunicación es un aliado importante en este aspecto.

En relación a la cantidad de pasta dental, menos del $50 \%$ de los entrevistados conocían que un exceso de flúor puede ser perjudicial y un menor porcentaje sabía sobre la cantidad de pasta que debe ser aplicado en el niño. En el estudio de Liu et al., el 64\% de padres y $68 \%$ de profesores conocían que un exceso de flúor podría ser perjudicial, cifras superiores a los que se encontró en este estudio, lo que podría deberse a la implementación de programas educativos de salud oral en China (Liu et al.), y que carece el Perú como práctica general en todas las escuelas, aún cuando existe la Estrategia Nacional de Salud Bucal del Ministerio de Salud. Sobre la cantidad de pasta dental, en el presente estudio los porcentajes de respuestas acertadas para los padres fueron inferiores a lo reportado en el estudio de Liu et al., (41\% y $48 \%$, respectivamente), mientras que fueron algo superiores en los profesores ( $46 \%$ y $42 \%$, respectivamente); sin embargo, difiere a lo encontrado por Gussy et al., donde un $75,7 \%$ de los padres recono- 
cían que la cantidad de pasta que debían emplear era el del tamaño de un guisante o menos. Los resultados de este estudio podrían estar indicando conductas peligrosas sobre el uso de las pastas dentales fluoradas en los infantes. Es aconsejable que los maestros, reconocidos como agentes importantes en la implantación de prácticas saludables en etapas tempranas, reciban capacitación en aspectos preventivos básicos para la salud bucal y general, como parte de su formación profesional.

Aún cuando el dentista fue el que suministró con mayor frecuencia la información sobre el flúor, otro estudio (Liu et al.), encontró que fueron los medios masivos. Dado que en esta pregunta se podía responder con más de una alternativa, si bien es cierto que más de la mitad de los profesores y padres recibieron la información del dentista, proporciones algo menores contestaron que también fueron informados por los medios masivos, lo que indica la alta influencia de los medios de comunicación para aspectos de conocimientos y posiblemente también de prácticas en salud oral.

Sin embargo, cuando se relacionó el nivel de conocimiento con la fuente de información, se encontró que la mejor fuente de información fue la charla educativa para el caso de los profesores. En los padres, además de esta, fue el propio dentista. Esto lleva a pensar que una mejor transmisión de información es del tipo presencial, como en el caso de charlas educativas y el dentista, donde puede interactuar el transmisor (profesional de la salud) con los receptores de la información (padres y profesores). Adicionalmente, el dentista y el responsable de la charla educativa tienen la información adecuada y tienen la capacidad de responder acertadamente a las preguntas que pudieran surgir en el proceso. Sin embargo, los medios masivos son los que transmiten la información a la mayoría de los padres y profesores, lo que lleva a concluir que debieran de incrementarse los programas educativos en salud oral por parte de los servicios de salud y los dentistas, a su vez, incrementar sus actividades educativas dentro del consultorio.

A pesar que las encuestas fueron anónimas, es posible, que en el caso de los padres, no se haya recogido la información exacta puesto que las encuestas fueron resueltas en los hogares y podrían haber recurrido a otras fuentes de información cercana antes de responder las preguntas. Otra limitación fue que, al ser auto aplicadas, no hubo la posibilidad de absolver las dudas que pudieron surgir al intentar contestar las preguntas ni de verificar el que hayan respondido todos los ítems.

Se concluye que el nivel predominante de conocimientos sobre pastas fluoradas en los padres y profesores fue el nivel medio y estuvo asociada al dentista y la charla educativa como fuentes principales de información; sin embargo se requieren reforzar aspectos como el del efecto perjudicial de su uso en exceso o en cantidades inadecuadas, a través de programas educativos en salud oral dirigidos a los padres y profesores. El papel del dentista es importante como ente promotor de la salud.

AGRADECIMIENTOS. Al Instituto de Investigación de la Facultad de Odontología de la Universidad de San Martín de Porres por el apoyo en el financiamiento del estudio. A las directoras y directores de las instituciones educativas y a los padres y profesores participantes en este estudio.

MATTOS-VELA, M. A.; CARRASCO-LOYOLA, M. B. \& VALDIVIA-PACHECO, S. G. Level of knowledge about fluoride toothpaste in parents and teachers from preschool children.Int. J. Odontostomat., 7(1):17-24, 2013.

ABSTRACT: The study aimed to determine the level of knowledge about fluoride toothpaste based on the fluoride source for parents and teachers from preschool children. A questionnaire was applied in a representative sample of parents and all teachers working at preschool educational state institutions in the Molina district, Lima, Perú. The level of knowledge was classified into three levels: low (0-2 points), medium (3-5 points) and high (6-7 points). We used frequency distribution tables for the univariate analisys, the chi square test and Fisher exact test for the bivariate analisys. The most frequent level of knowledge about fluoride toothpaste was medium level in both, parents $(n=68,45 \%)$ and teachers $(n=52,58.4 \%)$. The item: brushing teeth with fluoride toothpaste can cure the initial decay, obtained (had) the lowest number of correct response $50(33.1 \%)$ and $19(21.4 \%)$ parents and teachers respectively. It was found that the level of knowledge related to sources of information about fluoride is in the case of parents, educational talk $(p=0.014)$ and the dentist $(p=0.003)$ were associated, whereas in the case of teachers, only statistically significant association was found with the educational talk $(p=0.013)$. The higher level of knowledge about fluoride toothpaste on parents and teachers was the middle level and was associated with the dentist and educational talk as major sources of information.

KEY WORDS: dentifrices, fluorides, oral health. 


\section{REFERENCIAS BIBLIOGRÁFICAS}

Arblaster, L.; Lambert, M.; Entwistle, V.; Forster, M.; Fullerton, D.; Sheldon, T.; et al. A systematic review of the effectiveness of health service interventions aimed at reducing inequalities in health. J. Health Serv. Res. Policy, 1(2):93-103, 1996.

Barbería, E.; Cárdenas, D.; Suárez, M. \& Maroto, M. Fluoruros tópicos: Revisión sobre su toxicidad. Rev. Estomatol. Herediana, 15(1):86-92, 2005.

Contreras, N.; Valdivieso, M. \& Cabello, E. Nivel de conocimientos y prácticas de medidas preventivas de profesionales de salud sobre caries dental en el infante. Rev. Estomatol. Herediana, 18(1):29-34, 2008.

Crespo, M. I.; Riesgo, Y.; Laffita, Y.; Rodríguez, A. M. \& Copello, A. Instrucción educativa sobre salud bucal en la Escuela Primaria Lidia Doce Sánchez. Medisan, 14(2):232-42, 2010.

Delgado, E.; Sánchez, P. C. \& Bernabé, E. Mejora en los conocimientos, actitudes y prácticas a través de una intervención en salud oral basada en comunidad. Rev. Estomatol. Herediana, 16(2):83-8, 2006.

Forward, G. C. Action and interaction of fluoride in dentifrices. Community Dent. Oral Epidemiol., 8(5):257-66, 1980.

Gussy, M. G.; Waters, E. B.; Riggs, E. M.; Lo, S. K. \& Kilpatrick, N. M. Parental Knowledge, beliefs and behaviours for oral health of toddlers residing in rural Victoria. Aust. Dent. J., 53(1):52-60, 2008.

Liu, M.; Zhu, L.; Zhang, B. \& Petersen, P. E. Changing use and Knowledge of fluoride toothpaste by schoolchildren, parents and schoolteachers in Beijing, China. Int. Dent. J., 57(3):187-94, 2007

Lussi, A. Comparison different methods for the diagnosis of fissure caries without cavitation. Caries Res., 27(5):40916, 1993.

Margolis, P. A.; Stevens, R.; Bordley, W. C.; Stuart, J.; Harlan, C.; Keyes-Elstein, L., et al. From concept to application: the impact of a community-wide intervention to improve the delivery of preventive services to children. Pediatrics, 108(3):E42, 2001.

Meier, J. C. \& Jensen, M. E. Management of the questionable carious fissure: invasive vs. noninvasive techniques. $J$. Am. Dent. Assoc., 108(1):64-8, 1984.

Mellberg, J. R. Fluoride dentifrices: current status and prospects. Int. Dent. J., 41(1):9-16, 1991.
Ministerio de Salud. Prevalencia nacional de caries dental, fluorosis del esmalte y urgencia de tratamiento en escolares de 6 a 8, 10, 12 y 15 años, Perú. 2001-2002. Lima, Ministerio de Salud. Oficina General de Epidemiología y Dirección general de Salud de las Personas, 2005.

Pérez, A. G. ¿Es la Caries dental una enfermedad infecciosa y transmisible? Rev. Estomatol. Herediana, 19(2):11824, 2009.

Dirección para Correspondencia:

Manuel Antonio Mattos-Vela

Facultad de Odontología

Universidad de San Martín de Porres (USMP)

Lima

PERÚ

Email: manuel_mattos@yahoo.com

Recibido : 22-06-2012

Aceptado: 15-11-2012 